\title{
THE POLLICINA PROJECT: INTERWEAVING THE CULTURAL HERITAGE AND THE TERRITORY
}

\author{
Silvia Calegari, Floriana Meluso, Paolo Avogadro, Matteo Alessandro Dominoni \\ University of Milano-Bicocca, DISCo, Viale Sarca 336 Building 14, 20126 Milano, Italy
}

\begin{abstract}
The Pollicina project is aimed at fostering an interweaving between the educational context and the cultural heritage of the Lombardy region in Italy. The idea is to connect schools with museums, art locations etc., by guiding groups of students towards a collaborative creation of thematic cultural paths. From the end user point of view, Pollicina includes three main steps: the population of the databases, the creation of the cultural paths, and their fruition with the help of augmented reality solutions. From the structural point of view, the main feature of the e-blended platform (EduSN, Educational Social Network) associated with Pollicina, has been to design a database able to incorporate different knowledge sources and materials and to find solutions to connect them, passing from single entities to a collaborative system which evolves in time as more people access to the cultural heritage sites. In this respect, we present here the part of the structure of the database associated with the art objects/institutions and the information needed to create links among them.
\end{abstract}

\section{KEYWORDS}

Cultural Heritage, Thematic Cultural Path, Flipped Learning, Database

\section{INTRODUCTION AND DESCRIPTION OF POLLICINA}

The digitalization of art material and institutions, and in general the cultural heritage, is an active research field (F. Stanco, et al. 2011). The problems related with this subject include the heterogeneity of the material forms, the accessibility, the change in location and status of the material, etc. (Arizpe 2004). In the literature several methods to provide a digital version of the artworks which achieved a high degree of success have been proposed (Kreps 2003). A different side of the problem is related to the fruition of the cultural heritage. It should be noted that the digital formats are often built as complements and enhancements for the visit on site. In this respect, the amount of digital content of the art institutions is rapidly growing and calls for tools able to provide a better on site fruition of the material. On top of it, it is important to go beyond the single museum and provide connections among different institutions (i.e., schools, museums, and in general, art and culture institutions) and also with the territory. This can be seen as change of point of view, where the focus is no longer the single art object but there is an organic approach where the cultural background becomes an active player rather than just a static entity. The need is thus shifting towards finding better ways to transfer the cultural heritage to the users in an appealing and personalized way in order to form a higher degree of understanding and retention of the information. Pollicina is born within these premises (Calegari, et al. 2017), and it aims at connecting schools and the cultural heritage of region Lombardia. This is done by means of a collaborative environment which allows to coordinate the construction of "knowledge" paths. Furthermore, Pollicina allows to create a network which blends together famous cultural institutes and smaller entities. The main targets of the project are schools, museums, and in general, art and culture institutions. A strong motivation for this project is the observation according to which many of the institutions of a given region act as separate entities and separately they interact with those who are interested in visiting them. This lack of connection among the different institutions, is engrained in their very statuses: each museum has a focus and wants to follow it rather providing a general overview of all the other art/cultural sites; for example, a museum regarding paintings of the $18^{\text {th }}$ century has no direct connection with a collection of historic cars of a given brand. Nonetheless the very same people might visit both of the institutions, and in this respect, they turn out 
to be source of links between topics which might appear as completely uncorrelated. Instead of thinking in terms of what the single cultural institution can provide, it is possible to start from a completely different approach, where a theme is provided, and a cultural path among the different institutions is formed. From the perspective of a single visitor, however, it is difficult to find connections among different entities and material, while a collective approach can overcome this problem. Among the visitors, schools play a very important role, both from the numeric point of view, and because they also provide fresh minds in need of enriching their perspectives.

Pollicina is born to fill this knowledge gap. This project is structured in phases, where at the beginning the art institutions and the material need to be digitalized and inserted in a single comprehensive framework (Calegari, et al. 2018). This requires the collaboration of Pollicina staff with the institutions which provide material about their collections. An important percentage of the material of the museums had already been incorporated within a formal representation supported by the Lombardy region, according to which museums and art sites were supported to create digital cards in the SIRBeC standard (Sistema Informativo dei Beni Culturali della Regione Lombardia). An initial problem related to the population of the Pollicina database, regarded the fact that this standard has not been followed precisely by all the institutions, and in practice, each institution had modified versions of it. Moreover, this standard is not rich enough for the purposes of the project, in particular it does not include the perspectives of the visitors, but only the general information of the art object and its description. For these reasons we had to develop a new framework able to incorporate and standardize all the information needed by Pollicina.

This led to a data insertion phase which has been done in collaboration with the schools. It has to be noticed that, within the Italian high school system there is a directive called "Alternanza Scuola Lavoro", according to which, before the end of the learning cycle, the students must undergo a limited amount of hours in working contexts (this is done in order to connect the school and job world). In this respect, Pollicina provided a work opportunity for the students within a cultural context, where the job of the students was to enrich the information present on the cards. This process comprises the following steps: a student is assigned one or more cards regarding art objects, institutions, authors, etc. which are partially filled. Because of the diverse standards followed by each institution and the old cards contain less information than the one required by Pollicina, the students' job is to enrich (and complete) the cards using external knowledge sources like internet, books, etc. An important second step of this process involves on site visits, in which the students obtain further information by directly observing the object associated with the card. Pollicina provides a workflow regarding the data filling process in order to obtain high quality level for the cards of the database. Within this publication workflow three main actors play an important role: (1) the students, which collaborate to fill the cards but also provide a first level of critical review of their peers, (2) the teachers which review and can require modifications, and (3) the expert provided by the cultural institution owning the art material, who certifies (or rejects) the card under consideration. Although the information of the cultural institutions is extremely valuable (comprising of pictures, dates, descriptions, etc.), it requires further enrichment, since the aim of the project is to connect the artistic and historic background of the region, with the help of the visitors. In this respect, the new format stemming from the SIRBeC allows to incorporate personal notes and comments of the visitors. The core idea of this format is that the notes about the experiences allow to form new links among the objects and entities. The network among art objects and institutions formed becomes stronger and stronger as new notes and reports are included within the EduSN platform.

A core innovation of EduSN resides in providing a semantic structure able to contain different data levels. In particular, at the bottom level, there are the art objects: each object is contained within a space, which can be either an indoor or outdoor space, etc. These spaces provide a basic connection level among the art objects. The spaces are embedded within the art institutions which in turn follow a geo-localization system. The localization provides a geographical level of connection among the institutions, where close museums might share common historical happenings, etc. On the other hand, in order to provide stronger connections, there are two tools of EduSN: the navigation system and a clustering process which allows to handle the partial information related to the cards. The first one is based on the data of the cards and takes into account the link provided by the semantic structure: object, space, institution. The second one allows for an ever-growing set of links obtained with a clustering process based also on the storytelling of those who visited the institutions. Since the users can perform many different actions and the result of their work is going to influence the results of the paths, it is important to monitor their activity in order to guide them and to better model the platform for an improved fruition. In this respect, the next section introduces the soft skills which play an active role for Pollicina. 
It should be noticed that Pollicina is an ongoing project, that at present it includes 25 cultural entities, most of which being museums ("interactive museum of cinema MIC", "cultures museum Mudec", etc.) but there is also a presence of historical/architectural sites like the "Monastero di Torba" (Torba's monastery). Fourteen schools adhere to the project.

\section{AN OVERVIEW OF THE MAIN POLLICINA'S SERVICES}

EduSN is developed as a SaaS (Software as a Service) accessible by any devices (e.g., tablet, smartphone, laptop, etc.) and offers a great variety of social services designed to support learners during their cultural training. From an architectural point of view, thanks to the SaaS paradigm, each social service is defined as a microservice, namely an independent process that communicates with the device through "http API calls". The core set of the EduSN's services are:

- "Data Filling": this service is dedicated to the management of the cultural heritage materials provided by the cultural institutions. A first phase of digitalization of the material according to ad-hoc semantic representation (Calegari and Dominoni 2018) (Andrews and Higson 2008) is provided by the staff of Pollicina. Following this phase, the students can access to the cards of a specific cultural material, and enrich the content in a collaborative way, sharing comments and information. The final phase of this workflow regards a cultural expert (provided by each cultural institution), who has the task to certify the quality of the work.

- "Personalized Itineraries": this service is dedicated to the definition of customized itineraries aimed at connecting the aspect of the "Art Education" with the culture heritage territory. EduSN gives two possibilities for building the itineraries: ArtTour and Game, respectively.

$1 \quad$ In the "ArtTour" service, once a topic has been assigned by the teacher, the students are grouped into an editorial committee for the creation of the cultural path. This includes several phases: the mind map phase, where students share ideas, provide an outline of the concepts and select the cultural material; a collection phase, dedicated to the final selection of the cultural material and where students can enrich each material with multimedia information (such as video, external web links, images, documents, etc.); an itinerary phase, where the students write a textual description of the cultural path with the help of a collaborative and concurrent editor, and then they establish the order of the visit by using indoor maps for the definition of the relative logistic paths.

2 the "Game" service provides the possibility to select several games (e.g., puzzle, quiz, conceptual map) in order to help (younger) students to memorize art notions and concepts of specific material by accessing an amusing environment. With this service, a cultural itinerary becomes the gradual aggregation of several games. Once the itineraries have been defined, students can perform their visit to the indoor/outdoor cultural heritage via a dedicated App. With this App it is possible to realize an augmented visit by obtaining more information thanks to the use of the beacon technology and/or QR codes.

- "Magazine": it is dedicated to collect the most significant cultural paths (which later can be reused by other students, or in modified in order to produce new paths).

- "Dashboard": the whole set of activities performed by learners are monitored and analysed to define quantitative parameters that in a gamification perspective are converted into badges to identify the soft-skills of students.

The Pollicina's educational services are designed according to the guidelines of the flipped learning paradigm. This implies that a central attention has been paid to the collaborative aspects, i.e. how learners can improve their own skills by interacting with peers and experts anywhere and anytime. This is made possible by the adoption of virtual learning environments where learners and teachers can join and cooperate for a common goal beyond the limits of a traditional classroom. This collaborative aspect is functional in allowing the students to improve their self-esteem and the practices of learning. In this respect, EduSN allows the teachers to act as managers who oversee the student actions (editing, reviewing, etc.) by defining a methodology which allows to identify their soft-skills. Within a flipped learning process, the act of learning is, in fact, a social activity where students learn from each other by interacting and sharing their ideas and multimedia materials. Within a virtual learning environment, the actions among users are monitored and then analysed by using ad-hoc soft-skills. The Soft-skills are a combination of people skills, social skills, communication skills, character traits, attitudes, career attributes, social and emotional intelligence quotients among others (Avogadro, et al. 2016) (Keow Ngang, 2018) (Schulz, 2008) that enable the users to work well 
with others, perform well, and in general to produce a synergy which allows to achieve the given objectives. This general growing process could involve the acquisition of several abilities such as leadership, teamwork, problem solving, communication, interpersonal skills, etc. A proper identification of such skills can be viewed as a tool to improve the educative context by supporting the teachers for: reducing school dropping out, bridging the mismatch between training and labour market (thus promoting employability rates), suggesting career paths, etc. In this respect, the soft skills of the students can be considered as the glue which allows to connect different people and ideas, and as a result to spread information regarding different institutions. This in turn allows to strengthen the links between different art objects thus allowing to create a web of objects which are inter-correlated and naturally form the cultural paths.

\section{3. “CONVERSATION PRISM”AND SOFT-SKILLS IN POLLICINA}

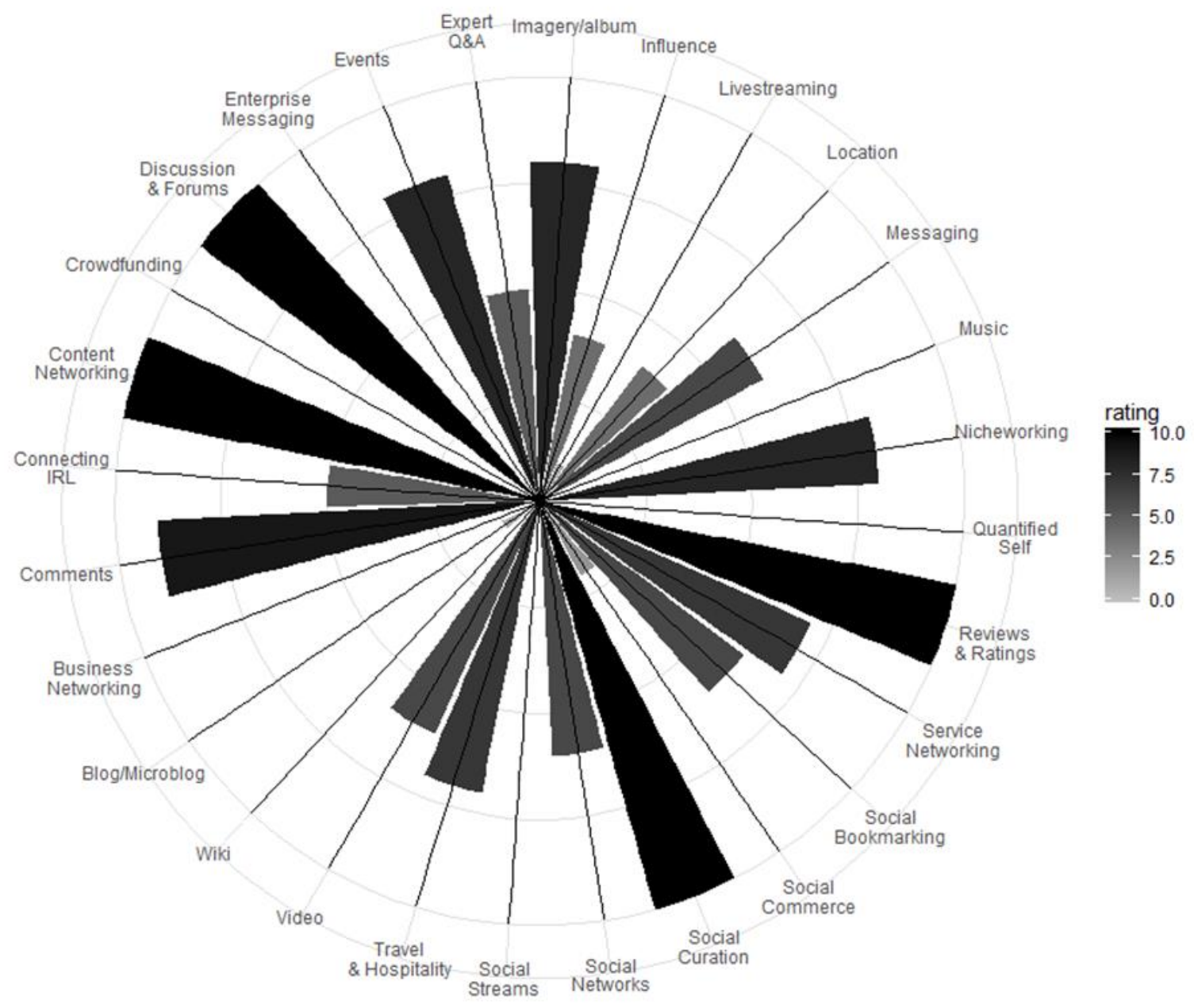

Figure 1. The "Conversation Prism" for the Pollicina project: Soft-SKILLs

A proper identification of the core set of soft-skills for the users of EduSN is based on the educational services that the platform can offer. This section is aimed at classifying the main services of Pollicina into the the-facto standard "conversation Prism" defined by B. Solis and J. Thomas (Solis, 2017) in order to have a comparison with the most popular social applications of the market. "A conversation prism is a graphic online chart of conversations between users which populate online communities and networks connected by a multitude of social media mediums and applications". 
Figure 1 shows the properties defining the "conversation Prism", and how the EduSN's services are classified according to it. This embryonic classification has been obtained by asking three educational experts involved in the project to express a grade on each of the possible features of the Pollicina project within the "conversation prism" and then averaging these values. This analysis has been done after each of the experts had utilized the prototype of EduSN. The values were ranging from 0 to 10 (where a low value indicates a poor connection to the property, and a high value indicates a strong connection to the property). For a better understanding, it is important to notice that the average grade associated with each property defines both the length and the color of the slices in the graph. In particular, the maximum length is related to a 10 and the black colour; while a 1 is associated with the smallest possible slice size with a light grey colour. Both the length and the colour scale linearly between the two extreme values.

The properties of the Pollicina project which obtained the highest scores are: "Discussion \& Forum", "Content Networking", "Comments", "Social Curation", "Reviews and Ratings", "Nicheworking", (i.e., a network of users with similar interests), "Imagery/album", and "Events". These dimensions reflect the educational motivations proper of Pollicina, where the collaborative aspect is a key element of the flipped learning. On the other hand of the spectrum the properties for which the lowest values have been obtained, includes: "Crowdfunding", "Business Networking", "Wiki", "Social Stream", "Quantified Self" (i.e., use of technology for data acquisition on aspects of a person's daily life), and "Music". The first two dimensions are not considered in Pollicina since this project is not aimed at a business audience, but it wants to be distributed to scholastic institutions and to be part of an open source community. Although EduSN allows learners to collect cultural data, at present no data streaming is involved and there is no possibility to create sound tracks; in addition, the sharing phase among learners is obtained by using comments, post-it notes, discussions and forums while at the moment no wiki has been implemented.

\section{POLLICINA DATABASE STRUCTURE}

The database underlying EduSN includes 13 macro areas which comprise 67 tables. In the following description we will provide an extract of the total database, which has been selected in order to provide the essential information that is being utilized for describing the different levels of granularity, from the objects to the institutions, but also including the part of the database which contains the information essential for creating connections among different objects (this includes the emotions and comments of the visitors).

In detail this restricted part of the database includes 5 macro areas and 25 tables.

The complexity and heterogeneity of the cultural heritages has led to the decision of exploiting a hybrid database that combines the "relational" and "semantic/schemaless" representation of EduSN. The data management plan was designed to take into account the storage aspects of the Pollicina platform. As mentioned before in Pollicina there are many different activities, this led to an enrichment of the fields of each cultural heritage card formatted according to the JSON standard. The database of Pollicina does not follow the classical model "Onthology + GraphDB". This is due to the heterogeneity of the data (paintings, sculptures, cars, planes, video, anagraphic profile of the schools joining the project, etc.), the management costs and the fact that it needs to keep an underlying structure (consisting of institutions, spaces, art objects) while the connections are expected to dynamically change according to the visits and the usage of the platform. In this respect, the users can add comments, emotions and social aspects related to the visit. 


\subsection{EER Diagram}

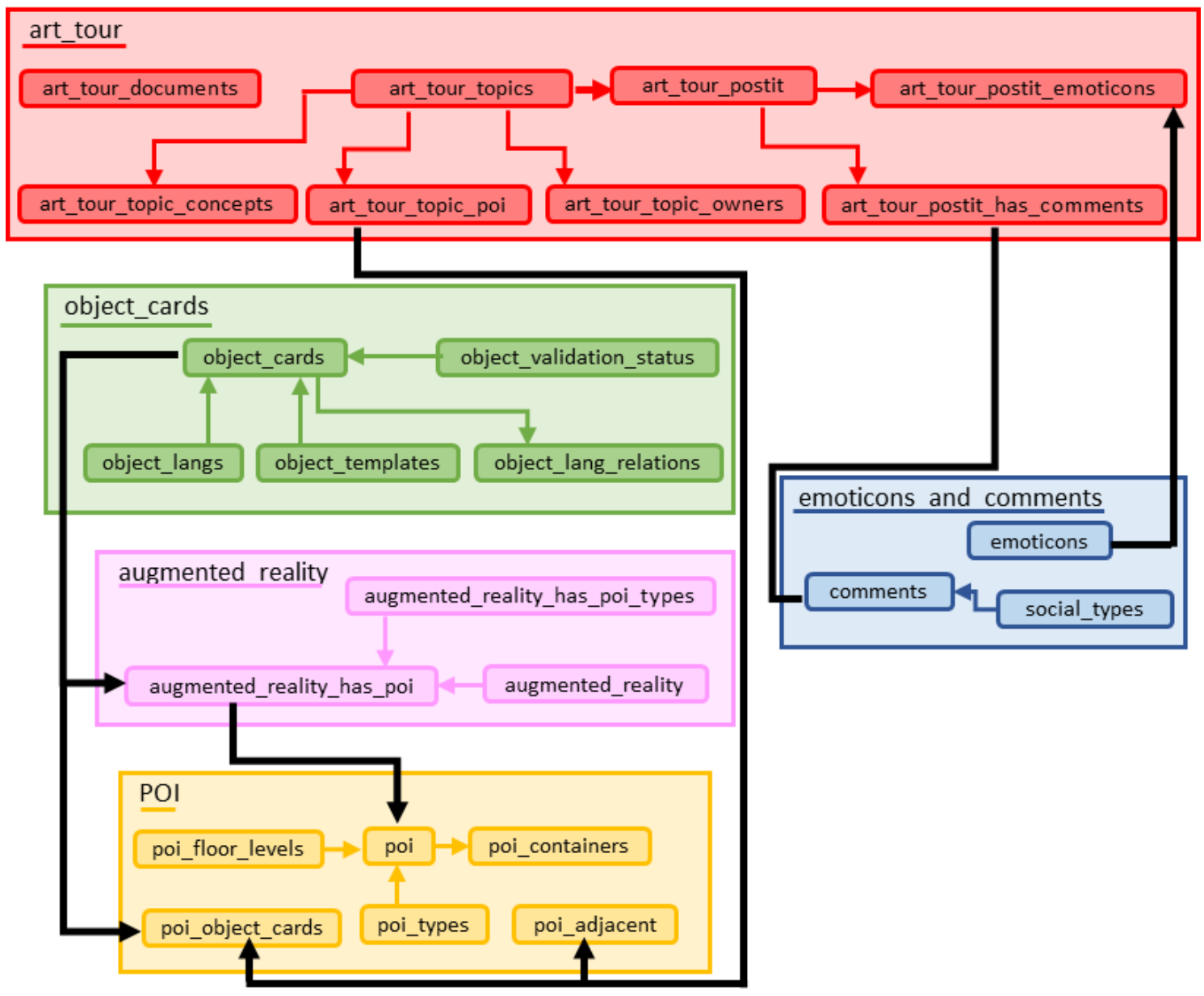

Figure 2. EER diagram of EduSN

This is a portion of the EER schema which describes the relations among the different entities. It is composed of 5 macro areas named: object_cards, POI, art_tour, augmented reality, and emoticons_and_comments. In the first phase of the project, the information given by the institutions has been digitalized and the cards, representing the cultural heritage object, created. These cards are stored in the macro area object_cards. The "Points Of Interest" (POI) are the institutions, their indoor rooms and outdoor spaces, which are included in the POI macro area. The core activity of the Pollicina project is the definition of the itineraries and art_tour is the macro area representing that. Once the itineraries are defined, the indoor visit can be performed with the help of a dedicated App that allows to realize an augmented visit present in the augmented reality macro area. Since the interaction among the users allows to create connections among the material, these interactions play a fundamental role for the project; for this reason, the macro area named emoticons_and_comments stores all the comments and emoticons generated during the art_tour phase.

In detail there are 5 macro areas:

1 Object_cards: this macro area contains all the tables linked to the object_cards. An object_card represents a single cultural heritage entity. A table with the same name of this macro area, stores all the cards (object_cards table). There are 4 types of object_cards (contained in the table object_templates): "OA" that stands for "Opera d'Arte" or art object; "RA" meaning "Reperto Archeologico" or archaeological find; "A" that is "Architettura" or institution and "AUT" is "Autore" or author. The data population phase represents the initial part in which the students need to complete the cards of the different objects. The workflow associated with a card publication is composed of different stages and statuses, these last ones are stored in the table object_validation_status. The table object_langs contains the different languages in which a card can be written. 
2 POI: As said before, "Point of Interest" includes cultural institutions, museums, and churches, etc. and for each of these institutions, indoor rooms and outdoor spaces are collected in the POI table. All the different objects (art object, archaelogical find, etc.) of a POI are stored in the poi_object_cards table. The table poi_containers includes only the rooms and spaces of the istitutions.

3 Art_tour: this macro area describes the core activity of the Pollicina project. A teacher defines a theme (stored in the art_tour_topics table) and some associated concepts (saved in art_tour_topic_concepts table). Since the teachers act as managers of the cultural path creation, the cards, of those who defined at least one theme are stored in the table art_tour_topic_owners. Once the cards are ready, the students can associate them to the concepts, represented by post-it notes within the frontend part of the platform (collected in the art_tour_postit table). It is important how the students collaborate with their peers, so the emoticons (allowing to like or dislike a post) and the comments are saved in two tables, respectively, art_tour_postit_emoticons and art_tour_postit_has_comments. The collaboration phase, where the students can communicate with each other, is named "collaborative editor" (table art_tour_documents).

4 Augmented reality: is the macro area related to the enhancements provided by Pollicina to the onsite visits. The students can, in fact, obtain more information thanks to the use of the beacon technology and/or QR codes. The institutions that enable the installation of these technologies are catalogued in the ar_has_poi table, while the ar_has_poi_types table collects the different types of tecnologies installed.

5 Emoticons_and_comments: the App used during the visit allows to store emotions, comments, etc. It is possible to like or dislike itineraries and this information is stored in the emoticons table, while all the comments are in the comments table. It should be remarked that the information here contained is not just frivolous, but it allows to add connections between different materials and institutions, and for this reason plays an important role in the project.

\section{CONCLUSIONS}

This paper presents the structure of the backend part of EduSN, the platform of the project Pollicina. This project allows to create cultural paths among different cultural institutions of the Lombardy region in Italy. In order to create paths connecting museums, churches, art collections etc., it is necessary, as a preliminary step, to populate the database of the project and, for this reason, about 400 high school students have been involved in the project as test users. The nature of the project follows the flipped learning paradigm where the interaction among the peers is essential. For this reason, the soft skills associated with the students become a pivotal part of the process and need to be monitored and understood. Once they are known it is in fact possible to use them for better balancing the groups, for suggesting roles and helping the students to grow in areas where they are not particularly strong. Unfortunately, there are many paradigms associated with the soft skills and the very concepts are difficult to define precisely. For this reason, we resorted at following the conversation prism which allows to better describe which are the most important aspects involved in the activities of Pollicina.

A complex part in the realization of this project, as an educational system, is related to providing a framework that can store heterogeneous formats and entities and which is able to find connections among the entities. This system needs to incorporate efficiently the material, but it must also provide a complete profiling of the actions of the users in order to define their soft-skills and the utilization patterns of the platform. On top of this, it has to be remarked that the connections among entities cannot be static, but they naturally need to evolve according to the new information produced by the onsite visits. For this purpose, it has been necessary to design a specific semantic structure allowing to comprise object cards, spaces, institutions, covering a wide range of formats. The connections among the entities are provided by two main technologies, one related with a navigations system based on the structure of the cards and the relations among them, while a second one, taking into account the feedbacks, storytelling and comments is a clustering procedure. We present here a detail of the EER schema of the database, in order to understand how the different phases of the project, stemming from the data insertion to the fruition of the cultural paths, are managed by the platform. 


\section{ACKNOWLEDGEMENT}

The Pollicina project is supported by the Regional Operational Program of the European Fund for Regional Development 2014 -2020 (POR FESR 2014-2020).

\section{REFERENCES}

J. Andrews, H. Higson, 2008 Graduate Employability, 'Soft Skills' Versus 'Hard' Business Knowledge: A European Study, Higher Education in Europe, 33:4, 411-422, DOI: 10.1080/03797720802522627

L. Arizpe, 2004, Intangible Cultural Heritage, Diversity and Coherence, Museum International, Volume56, Issue1-2, May 2004, Pages 130-136, Wiley Online Library.

S. Calegari, F. Meluso, G. Saudati, M. Dominoni. The "Pollicina" Project: A Social Learning Management System in The Cultural Heritage Domain. 8th International Conference the Future of Education, Florence Italy, 8-9 June, Edited by Pixel, ISBN: 978-88-3359-020-2, 2018, pp. 18-22.

S. Calegari, F. Meluso, P. Avogadro, M. Dominoni. A navigator for sharing Cultural Heritages in an educational context: the "Pollicina" project. 7th International Conference the Future of Education, Florence Italy, 8-9 June, Edited by Pixel, ISBN:978-88-6292-868-7, 2017, pp. 16-20

S. Calegari, M. Dominoni. The Pollicina Project a Collaborative and Educational Social Suite to Build Cultural Itineraries. \#EARTH2018 Digital Environments for Education, Arts and Heritage International and Interdisciplinary Conference Brixen (Extended Version), Italy 5-6/7, pp. 1-10. 2018. In press

P. Avogadro, S. Calegari, M. A. Dominoni, (2016) "Expert students in social learning management systems", Interactive Technology and Smart Education, Vol. 13 Issue: 3, pp.202-217, https://doi.org/10.1108/ITSE-06-2016-0018

T. Keow Ngang, 2018, The importance of soft skills acquisition by teachers in higher education institutions, Kasetsart Journal of Social Sciences, ISSN 2452-3151

C.F. Kreps, 2003, Liberating Culture, by Routledge 270 Madison Ave, New York NY 10016, USA.

B. Schulz, 2008, The importance of soft skills: Education beyond academic knowledge. Journal of Language \& Communication, 2(1), 146-154.

B. Solis, and J. Thomas, 2017 url:https://www.briansolis.com/2017/09/travel-vertical-brian-solis-jesse-thomas-capturestate-social-media-single-infographic/

F. Stanco, S. Battiato, S. Gallo, 2011, Digital imaging for cultural heritage preservation: Analysis, restoration, and reconstruction of ancient artworks. CRC Press, Taylor \& Francis Group,6000 Broken Sound Parkway NW, Boca Raton, Fl, USA 